\title{
Teamlets in Primary Care: Enhancing the Patient and Clinician Experience
}

\author{
Thomas Bodenheimer, MD, and Rachel Willard-Grace, MPH
}

Many primary care practices have created a team structure in which a clinician and medical assistant "teamlet" form the core of a larger team. The larger team comprises a few teamlets supported by other clinical personnel. Patients are empaneled to a particular teamlet. The teamlet structure, which turns large practices into small units, is attractive to patients, most of whom prefer small rather than large practices. Clinicians working in stable teamlets, with the same medical assistant every day, have less burnout than clinicians working with different medical assistants on different days. The teamlet model can thus create positive experiences for clinicians and patients alike. (J Am Board Fam Med 2016;29: 135-138.)

Keywords: Health Care Team, Primary Health Care

Primary care faces a series of dilemmas. Many patients face difficulty gaining prompt access to care because the number of primary care physicians is insufficient to meet the population's demand for care. ${ }^{1}$ Yet physicians face high levels of burnout and cannot add additional capacity. ${ }^{2}$ Primary care teams that empower all team members to take over portions of patients' care may add capacity without increasing physician stress. ${ }^{3}$ However, patients desire continuity of care and a trusting relationship with a personal primary care physician, goals that teams must try to provide. Primary care is challenged to achieve all 3 goals of prompt access; continuous, trusting relationships with patients; and physician well-being. This commentary explores whether teams can be structured to simultaneously add capacity, provide continuity of care, and reduce physician burnout.

This article was externally peer reviewed.

Submitted 26 May 2015; revised 31 August 2015; accepted 9 September 2015.

From the Center for Excellence in Primary Care, University of California, San Francisco.

Funding: none.

Conflict of interest: none declared.

Corresponding author: Thomas Bodenheimer, MD, Center for Excellence in Primary Care, Department of Family and Community Medicine, University of California at San Francisco, Bldg 80-83, SF General Hospital, 995 Potrero Ave, San Francisco CA 94110 (E-mail: TBodenheimer@fcm. ucsf.edu or tbodie@earthlink.net).

\section{Adding Capacity}

The primary care physician shortage is expected to reach 33,000 by $2035,{ }^{1}$ creating a large gap between population demand and primary care capacity. The gap is narrowed by the numbers of nurse practitioners and physician assistants, but still remains large and growing. ${ }^{4}$ High-functioning teams can reduce the demand-capacity gap. Registered nurses (RNs) and pharmacists, under standing orders, can independently provide high-quality care for patients with chronic conditions such as diabetes and hypertension, including the adjustment of medications. ${ }^{3}$ RNs can independently care for uncomplicated urinary tract infections, respiratory infections, and low-back pain without physician involvement, thereby adding capacity to see more patients without taking physician time. ${ }^{3}$ Medical assistants (MAs) working as "scribes" freed up the documentation burden in one study, saving primary care physicians 75 minutes during each 4-hour clinic session, thereby allowing physicians to see more patients, possibly without adding stress. $^{5}$

\section{Continuity of Care and Trust}

Studies suggest that patients want at least 4 things from their physician ${ }^{6,7}$ :

- Competence: I want my physician to have the knowledge necessary to help me. 
- Empathy: I want my physician to care about me.

- Familiarity: I want to know my physician and I want my physician to know me.

- Continuity: I want to see my personal physician when I need help.

The relationship over time-continuity of careallows patients and physicians to get to know one another and builds patients' trust that their physician is competent and caring. In addition, continuity of care is associated with higher patient and physician satisfaction, better preventive and chronic care outcomes, and lower costs. ${ }^{8}$ Patient trust in physicians leads to increased adherence to treatment recommendations and more healthy behaviors.'

It may be possible to extend patients' wishes for continuity, trust, and familiarity from a single physician to a team if the team is small. Patients prefer small over large practices. In a landmark study of 367 practices, patients were asked immediately following a physician visit: "How was your visit?" In solo practices, $64 \%$ rated the visit as excellent, compared with $48 \%$ in large practices $(P=.001) .{ }^{10}$ This preference is rooted in patients' desire to know who is caring for them and for that person/ team to know them. ${ }^{7}$ It is unreasonable to ask patients to transfer a trusting relationship with a physician to a large team with many members. Moreover, compared with a team of 2 physicians, a large team would be responsible for a big panel, making it impossible for all physicians to know and develop trusting relationships with all patients.

Patients can develop longitudinal trusting relationships with all team members. Compared with care by physicians, patients receiving care from $\mathrm{RNs}$ for uncomplicated health issues experienced greater satisfaction. ${ }^{3}$ Patients have greater trust in physical therapists than in physicians for management of low-back pain. ${ }^{3}$ Patients develop trust in pharmacists over time when they get to know the pharmacist and the pharmacist gets to know them. ${ }^{11}$ MAs are ubiquitous in primary care practices. In anecdotal evidence from visits to $>50$ primary care practices, we and our colleagues have heard from MAs and other team members that patients often develop longitudinal, trusting relationships with an MA who the patients know and who knows them. Moreover, a patient's trust in their primary care physician increases when the patient enters into close relationships with MAs who work with the patient's physician. ${ }^{12}$

A recent California survey found that lowerincome patients experiencing team-based care were significantly more satisfied than those without teams. In addition, $81 \%$ of those surveyed reported a willingness to be cared for by a team, even if that means seeing their physician less often. ${ }^{13}$ In other studies, patient satisfaction with on-team visits is high when the team is visible and its members know the patients well, explain things well, and coordinate care with the patient's personal physician. ${ }^{7,14,15}$

These conditions - teams being visible and team members and patients being familiar with one another-are best met if the teams are small and stable. Team stability means:

- members of the team always work together.

- patients are empaneled to a team.

- members of the team always care for patients on their team panel.

- patients receive all care from their team.

Summarizing to this point, evidence suggests that patients can transfer continuous, trusting relationships from single physicians to small, visible teams. Small practices offer small visible teams, but in 2011, 40\% of primary care physicians worked in groups with $>50$ physicians, and only $24 \%$ were in solo practice. ${ }^{16}$ Can larger practices organize themselves into small visible teams? Enter the teamlet.

\section{The Teamlet Structure}

A teamlet is a small team, often consisting of a clinician (physician, nurse practitioner, or physician assistant) who always works with the same MA in caring for a defined patient panel. ${ }^{17}$ Solo practices consist of 1 teamlet; practices with 2 clinicians may have 2 teamlets.

In larger practices, teamlets may constitute the core of a larger team. As pictured in Figure 1, the same clinician works with the same MA every day, and 3 teamlets are supported by such personnel as an $\mathrm{RN}$, behavioral health provider, pharmacist, health coach, and/or patient navigator. Patients requiring services that the teamlet is unable to provide receive additional care from the appropriate member of the larger team. 
Figure 1. One team, three teamlets. MA, medical assistant.

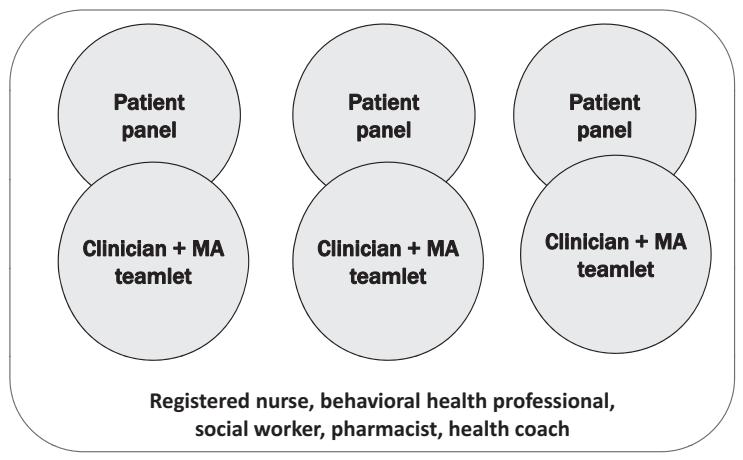

The teamlet model divides large practices into small units that, from the patient's perspective, resemble a small practice. Teamlets can transform a large, impersonal institution into a small practice environment within which patients know their teamlet members and the teamlet partners know their patients. To make teamlets visible, some practices provide patients with business cards showing the names, pictures, and contact information of teamlet members. ${ }^{18}$

In visits to many primary care practices, we observed that while most practices create teams and teamlets on paper, scheduling convenience often trumps team stability. In these cases clinicians do not always work with the same MA, and patients may receive care away from their home teamlet, thus disrupting the clinician-MA and patientteamlet relationships.

Many variations on the teamlet theme can be found in primary care practices. Two-person teamlets are limited in their ability to expand MA roles. Thus a teamlet might include 1 clinician and 2 MAs, allowing the MAs to take on panel management, health coaching, and/or scribe responsibilities. ${ }^{19}$ The Veterans Administration 4-person teams, called teamlets, include a clinician, RN, licensed practical nurse/licensed vocational nurse, and clerk (receptionist). ${ }^{20}$ In other practices a teamlet might involve 2 half-time clinician partners-one working Monday, Wednesday morning, and Thursday, and the other working Tuesday, Wednesday afternoon, and Friday - and 1 full-time MA. Patients are empaneled to one of the clinicians, but patients needing more frequent care come to know and be known by the other clinician. Practices invent other permutations of the teamlet model to fit staffing realities. Yet certain features are essential: the teamlet is small, forms the core of a larger team, is stable and visible, and cares for a defined patient panel.

\section{Team Function}

Team structure is but one element of team formation; a collaborative team culture is equally important. ${ }^{21}$ While many clinician-MA teamlets develop mutual trusting relationships, others are less collaborative. ${ }^{22}$ Some clinicians are difficult to work with, and some MAs are unenthusiastic about their work. We have observed practice leaders paying close attention to clinician/MA pairings to maximize collaborative team culture. ${ }^{23}$

\section{Teamlets and Physician Burnout}

In addition to offering patients longitudinal trusting relationships, can teams with a stable structure and collaborative culture reduce clinician burnout? A recent survey of 231 clinicians in 16 primary care practices found that, if a collaborative culture exists, clinicians working in a stable teamlet structure (regularly paired with the same MA) have lower levels of emotional exhaustion (a component of burnout) than clinicians working with different MAs on different days. ${ }^{24}$

\section{Conclusion}

It seems that small, visible primary care teamlets, working within larger teams, have the potential to reduce the primary care demand-capacity gap and relieve clinician burnout by adding capacity without increasing clinician stress. Simultaneously, patients may develop continuous, trusting relationships with members of the team. In a health system evolving toward large and impersonal practices, team-based care and the stable teamlet model may help to create a safe haven for patients and clinicians alike.

\section{References}

1. Petterson SM, Liaw WR, Tran C, Bazemore AW. Estimating the residency expansion required to avoid projected primary care physician shortages by 2035 . Ann Fam Med 2015;13:107-14.

2. Bodenheimer T, Sinsky C. From triple to quadruple aim: care of the patient requires care of the provider. Ann Fam Med 2014;12:573-6.

3. Bodenheimer TS, Smith MD. Primary care: proposed solutions to the physician shortage without training more physicians. Health Aff (Millwood) 2013;32:1881-6. 
4. Bodenheimer T, Chen E, Bennett HD. Confronting the growing burden of chronic disease: can the U.S. health care workforce do the job? Health Aff (Millwood) 2009;28:64-74.

5. Reuben DB, Knudsen J, Senelick W, Glazier E, Koretz BK. The effect of a physician partner program on physician efficiency and patient satisfaction. JAMA Intern Med 2014;174:1190-3.

6. Detsky AS. What patients really want from health care. JAMA 2011;306:2500-1.

7. Safran DG. Defining the future of primary care: what can we learn from patients? Ann Intern Med 2003;138:248-55.

8. Saultz JW, Lochner J. Interpersonal continuity of care and care out-comes: a critical review. Ann Fam Med 2005;3:159-66.

9. Thom D, Hall M, Pawlson L. Measuring patients' trust in physicians when assessing quality of care. Health Aff (Millwood) 2004;23:124-32.

10. Rubin HR, Gandek B, Rogers WH, et al. Patients' ratings of outpatient visits in different practice settings. Results from the Medical Outcomes Study. JAMA 1993;270:835-40.

11. McCullough MB, Petrakis BA, Gillespie C, et al. Knowing the patient: a qualitative study on caretaking and the clinical pharmacist-patient relationship. Res Social Admin Pharm 2015 Apr 27 [Epub ahead of print].

12. Thom DH, Hessler D, Willard-Grace R, et al. Does health coaching change patients' trust in their primary care provider? Patient Educ Couns 2014;96: 135-8.

13. Blue Shield of California Foundation. Connectedness and continuity: patient-provider relationships among low-income Californians. June 2012. Available from: http://www.blueshieldcafoundation.org/ sites/default/files/u14/BSCF_Patient_Provider_web. pdf. Accessed November 17, 2015.

14. Wagner EH. Are continuity of care and teamwork incompatible? Med Care 2007;45:6-7.
15. Rodriguez HP, Rogers WH, Marshall RE, Safran DG. Multidisciplinary primary care teams: effects on the quality of clinician-patient interactions and organizational features of care. Med Care 2007;45: $19-27$.

16. Welch WP, Cuellar AE, Stearns SC, Bindman AB. Proportion of physicians in large group practices continued to grow in 2009-11. Health Aff (Millwood) 2013;32:1659-66.

17. Bodenheimer T, Laing BY. The teamlet model of primary care. Ann Fam Med 2007;5:457-61.

18. Willard R, Bodenheimer T. The building blocks of high-performing primary care: lessons from the field. Oakland: California HealthCare Foundation; 2012. Available from: http://www.chcf.org/publications/ 2012/04/building-blocks-primary-care\#ixzz3k9n1DiKA. Accessed November 17, 2015.

19. Bodenheimer T, Willard-Grace R, Ghorob A. Expanding the role of medical assistants: who does what in primary care? JAMA Intern Med 2014;174: 1025-6.

20. Rosland AM, Nelson K, Sun H, et al. The patientcentered medical home in the Veterans Health Administration. Am J Manage Care 2013;19:e263-72.

21. Ghorob A, Bodenheimer T. Building teams in primary care: a practical guide. Fam Syst Health 2015; 33:182-92.

22. Elder NC, Jacobson CJ, Bolon SK, et al. Patterns of relating between physicians and medical assistants in small family medicine offices. Ann Fam Med 2014; 12:150-7.

23. Bodenheimer T, Ghorob A, Willard-Grace R, Grumbach K. The 10 building blocks of highperforming primary care. Ann Fam Med 2014;12: $166-71$.

24. Willard-Grace R, Hessler D, Rogers E, et al. Team structure and culture are associated with lower burnout in primary care. J Am Board Fam Med 2014;27: 229-38. 